\title{
Efecto del almacenamiento sobre la viabilidad y germinación de dos especies arbóreas tropicales
}

\section{Effect of storage on the viability and germination of two tropical tree species}

\author{
Carlos Alberto Ríos-García ${ }^{1 *}$, Carolina Orantes-García ${ }^{1}$, Rubén Antonio Moreno-Moreno ${ }^{2}$, Óscar \\ Farrera-Sarmiento ${ }^{1}, 3$ \\ ${ }^{1}$ Instituto de Ciencias Biológicas. Universidad de Ciencias y Artes de Chiapas, Libramiento Norte Poniente número 1155, \\ Colonia Lajas Maciel, CP. 29039, Chiapas, México. \\ ${ }^{2}$ Facultad de Ciencias Humanas y Sociales. Universidad de Ciencias y Artes de Chiapas, Libramiento Norte Poniente número \\ 1155, Colonia Lajas Maciel, CP. 29039, Chiapas, México. \\ ${ }^{3}$ Jardín botánico Faustino Miranda. Calzada de los Hombres llustres, Parque Madero, edificio Museo Botánico s/n, colonia \\ Centro Tuxtla Gutiérrez, Chiapas, México. \\ *Autor de correspondencia: carlos_garcia2009@hotmail.com
}

Nota científica recibida: 12 de Julio de 2016 aceptada: 15 de junio de 2017

RESUMEN. Cochlospermum vitifolium (Willd.) Spreng. y Quararibea funebris (La Llave) Vischer, son árboles tropicales nativos de México con importancia ecológica, social y económica; de los que no se tiene una práctica sustentable para el manejo. El objetivo fue evaluar la viabilidad y la germinación en semillas almacenadas a 0, 3, 6, 9 y 12 meses. Las semillas a los 0 meses de almacenamiento fueron viables en un 99.66 y $99.33 \%$ para C. vitifolium y $Q$. Funebris, respectivamente, mientras que a los 12 meses se tuvo disminución de la viabilidad, presentando una germinación final del $71.3 \%$ para C. vitifolium y $61.3 \%$ para $Q$. Funebris.

Palabras clave: Cochlospermum vitifolium, Quararibea funebris, Reserva de la Biosfera Selva el Ocote, Chiapas, recurso florístico

ABSTRACT. Cochlospermum vitifolium (Willd.) Spreng. and Quararibea funebris (La Llave) Vischer are tropical trees native to Mexico with ecological, social and economic importance; however, there is no sustainable management practice for these species. The objective was to evaluate viability and germination in seeds stored at $0,3,6,9$ and 12 months. The seeds at 0 months of storage were 99.66 and $99.33 \%$ viable for $C$. vitifolium and Q. Funebris, respectively, while at 12 months there was a decrease in viability, exhibiting a final germination of $71.3 \%$ for $C$. vitifolium and $61.3 \%$ for Q. Funebris.

Key words: Cochlospermum vitifolium, Quararibea funebris, Selva el Ocote Biosphere Reserve, Chiapas, floristic resource

\section{INTRODUCCIÓN}

La zona intertropical alberga la mayor biodiversidad del mundo para casi todos los grupos de organismos, y contribuye de forma importante en la riqueza biológica del planeta (Gaston 2000). En México, la diversidad biológica terrestre comprende una gran variedad de paisajes y de comunidades vegetales que cubren el territorio nacional (Challenger y Soberón 2008), de los cuales, los bosques tropicales húmedos albergan más de 5000 especies de plantas (Rzedowski 1991). Las cuales representan más del $80 \%$ de la flora arbórea nacional, ocupando el estado de Chiapas el segundo lugar después de Oaxaca (INEGI 2013). Ante el deterioro de este tipo de ecosistema, por el cambio de uso de suelo, la tala de árboles y la urbanización, se requiere conservar las especies nativas (Velázquez et al. 2002). Una forma es mediante el manejo de semillas o conservación ex situ, que se lleva a cabo en los bancos de germoplasma (Gentil 2001). Para ello es importante considerar el tipo de semillas a 
almacenar (Kameswara-Rao et al. 2007).

Las semillas ortodoxas, son aquellas que toleran la deshidratación, hasta niveles de humedad menores del $5 \%$ y las semillas recalcitrantes toleran la deshidratación entre 15 y $50 \%$ de humedad (Gentil 2001, Nkang 2002, Sánchez-Arellano et al. 2011). Dada la importancia de estos aspectos en el ámbito de la fisiología y tecnología de semillas, se han desarrollado diferentes protocolos para evaluar la viabilidad y vigor de las semillas, así como para lograr condiciones de almacenamiento que aseguren una mayor longevidad (Pérez-García y Pita-Villamil 2001). Para conocer este valor de viabilidad o capacidad de vida de las semillas, una de las pruebas utilizadas es la del tetrazolio (cloruro de 2, 3, 5, - trifenil-tetrazolio) (ISTA 2010). La cual se basa en la actividad de las enzimas deshidrogenasas, que catalizan las reacciones respiratorias en las mitocondrias, durante la glicólisis y el ciclo de Krebs (Franca-Neto et al. 1998), tiñendo los tejidos de rojo en regiones como la radícula, plúmula, eje embrional y cotiledones (Sánchez-Arellano et al. 2011).

Las especies Cochlospermum vitifolium (Willd.) Spreng y Quararibea funebris (La Llave) Vischer, son nativas de la Selva Zoque de Chiapas, las cuales son importantes en el aprovechamiento maderable para la construcción, artesanias y para la elaboración de utensilios y herramientas de trabajo (Pennington y Sarukhán 2005). Los registros indican que $C$. vitifolium presenta uso medicinal contra la diabetes y hepatitis, como los más frecuentes (Cedano-Maldonado y Villaseñor-Ibarra 2004). Mientras que las flores de $Q$. funebris, se usan como aromatizantes de bebidas como el pozol (Pennington y Sarukhán 2005). Por lo anterior, el objetivo del estudio fue evaluar la viabilidad y germinación de $C$. vitifolium y $Q$. funebris sometidas a diversos periodos de almacenamiento.

\section{MATERIALES Y MÉTODOS}

En el mes de marzo se recolectaron 2000 semillas de Cochlospermum vitifolium y Quararibea funebris de cinco árboles de cada especie, ubicados en un cuadrante de una hectárea, de la colonia agrícola General Cárdenas del municipio de Cintalapa, Chiapas, México. Que se localiza entre los $16^{\circ} 53^{\prime}$ $40^{\prime \prime}$ LN y $93^{\circ} 43^{\prime} 45.8^{\prime \prime} \mathrm{LO}$, a una altitud de $800 \mathrm{~m}$, tiene un clima cálido subhúmedo con lluvias abundantes en verano (Aw1), precipitación anual de 1 500 a $2500 \mathrm{~mm}$ y temperatura media anual de 22 ${ }^{\circ} \mathrm{C}$. Las semillas se separaron de forma manual y se almacenaron a temperatura ambiente $\left(25^{\circ} \mathrm{C}\right)$ en botes de plásticos transparentes libres de humedad.

\section{Viabilidad}

La viabilidad de las semillas se determinó a los $0,3,6,9$ y 12 meses de almacenamiento, mediante la prueba de tetrazolio (ISTA 2005), que consistió en remojar las semillas por $24 \mathrm{~h}$ para facilitar un corte longitudinal en la testa y separar los cotiledones, que se colocaron en cajas Petri a las que se les pusieron tres gotas de la solución de tinción de tetrazolio $(0.1 \%$ cloruro $2,3,5$ trifenil$2 \mathrm{H}$ tetrazolio-Sigma $\left.{ }^{\circledR}\right)$, las cuales se sometieron a oscuridad a $25{ }^{\circ} \mathrm{C}$ por $24 \mathrm{~h}$. En cada periodo de almacenamiento se utilizaron 100 semillas por especie con tres repeticiones. Las observaciones se realizaron con un microscopio estereoscópico Leica Zoom $200{ }^{\circledR}$, considerando el número de semillas basado en el color adquirido por los embriones, principalmente aquellos que presentaron un color rojo intenso (vigorosos), excluyendo los que tenían un color ligeramente pálido o no tenían color, que se consideraron no viables o muertos (Maldonado et al. 2016). El porcentaje de viabilidad se obtuvo con la siguiente formula:

$$
\% \text { Viabilidad }=\frac{\text { Semillas teñidas }}{\text { Semillas totales }} \times 100
$$

\section{Germinación}

Las semillas se desinfectaron en una solución al $1 \%$ de Agromycin 500 de Pfizer ${ }^{\circledR}$ (estreptomicina+oxitetraciclina+sulfato tribásico de cobre), durante 20 min en agitación constante, para luego lavarlas tres veces con agua destilada estéril. Para después colocar las semillas en charolas de plástico con peat moss esterilizado como sustrato. Se incubaron en una cámara bioclimática 
a $25{ }^{\circ} \mathrm{C} \pm 2{ }^{\circ} \mathrm{C}$ de temperatura, con fotoperiodo controlado de $16 / 8 \mathrm{~h}$ luz/oscuridad, con intensidad lumínica de 1800 lux. Se aplicó riego con agua destilada estéril a capacidad de campo cada tercer día. Las observaciones se realizaron cada $5 \mathrm{~d}$ durante 60 d. Se consideraron germinadas las semillas cuando presentaron emergencia sobre el sustrato (Hartmann y Kester 2001). Se utilizó un diseño completamente al azar con tres repeticiones de 50 semillas cada uno, para los tratamientos de las semillas almacenadas a 0,6 y 12 meses. Las variables evaluadas fueron las siguientes (González-Zertuche y Orozco-Segovia 1996):

Tiempo promedio de germinación ( $T$ ), como el tiempo promedio que necesitan las semillas para germinar

$$
T=\frac{\sum(n i t i)}{\sum n i}
$$

Donde: $\mathrm{ni}=$ número de semillas germinadas en el día i, y ti= tiempo de germinación desde la siembra hasta la germinación de la última semilla.

Índice de germinación (IG), que es afectado por la capacidad de germinación y no proporciona información acerca de la distribución de los eventos de la germinación en el tiempo.

$$
I G=\frac{\sum(n i t i)}{N}
$$

Dónde: $\mathrm{N}=$ Total de semillas sembradas

Velocidad de germinación (MG), que relaciona el número de semillas germinadas con el tiempo de germinación.

$$
T=\sum\left(\frac{n i}{t i}\right)
$$

Coeficiente de germinación (CV), basado en el número de semillas germinadas y se relaciona de forma inversa con el tiempo y el número de semillas germinadas por día.

$$
P G=\frac{\sum n i}{\left(\sum n i t i\right)} x 100
$$

Porcentaje de germinación final (PG), Para determinar el efecto del almacenamiento sobre la capacidad germinativa de las semillas.

$$
P G=\frac{n i}{N} x 100
$$

Germinación acumulada (GA), que muestra la forma en que se incrementa la germinación y el tiempo (días) de inicio de la germinación.

$$
G A=\frac{(\% n 1+\% n 2+\% n 3+\% n x)}{n x}
$$

Donde: $\% \mathrm{n} 1=$ porcentaje de semillas germinadas en el tiempo $1, \% \mathrm{n} 2=$ porcentaje de semillas germinadas en el tiempo $2, \% \mathrm{n} 3=$ porcentaje de semillas germinadas en el tiempo 3 y $n x=$ tiempo en que se presentó la germinación.

\section{Análisis estadístico}

Para el análisis de viabilidad y germinación, se realizó la prueba de Kruskal-Wallis y para evaluar el efecto del almacenamiento en la viabilidad y germinación de las semillas se realizó la prueba $U$ de Mann-Whitney usando el ajuste de Bonferroni con el paquete estadístico $R$ 3.2.3 ${ }^{\circledR}$.

\section{RESULTADOS Y DISCUSIÓN}

La viabilidad en ambas especies está influenciada por el tiempo de almacenamiento (Tabla 1). El promedio de viabilidad fue de 99.66 a $99.33 \%$ para Cochlospermum vitifolium y Quararibea funebris a los cero meses de almacenamiento, mientras que a los 12 meses la viabilidad disminuyó a 72.33 y $71.33 \%$, respectivamente; con diferencias estadísticas entre la viabilidad y el tiempo de almacenamiento $(p<0.0001)$ de las dos especies (Figura 1A y 1B). Esto puede estar relacionado con lo expuesto por Bidwell (2000) y Hartmann y Kester (2001) quienes indican que el envejecimiento es un factor que disminuye la viabilidad en las semillas, mientras que Ferguson (1995) y Ríos-García et al. (2016) menciona que la calidad de las semillas disminuye con el transcurso del tiempo, lo que ocasiona un deterioro que depende de las condiciones ambien- 
Tabla 1. Porcentaje de viabilidad por especies sometidas a almacenamiento, prueba de Mann-Whitney.

\begin{tabular}{ccc}
\hline & Cochlospermum vitifolium & Quararibea funebris \\
\hline Mes de & Porcentaje de viabilidad & Porcentaje de viabilidad \\
almacenamiento & y prueba de Mann-Whitney & y prueba de Mann-Whitney \\
0 & $99.66^{a}$ & $99.33^{a}$ \\
3 & $98.66^{a}$ & $98.33^{a}$ \\
6 & $98.66^{a}$ & $87.66^{b}$ \\
9 & $84.33^{b}$ & $80.66^{c}$ \\
12 & $72.33^{c}$ & $71.33^{d}$ \\
\hline
\end{tabular}

*Las letras representan las diferencias entre meses por cada especie.

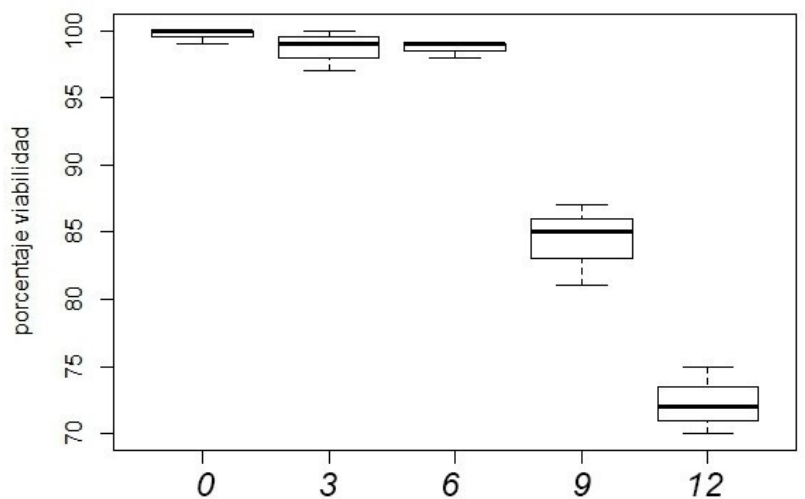

A)

Figura 1. Porcentaje de viabilidad por meses de almacenamiento A) C. vitifolium y B) Q. funebris.

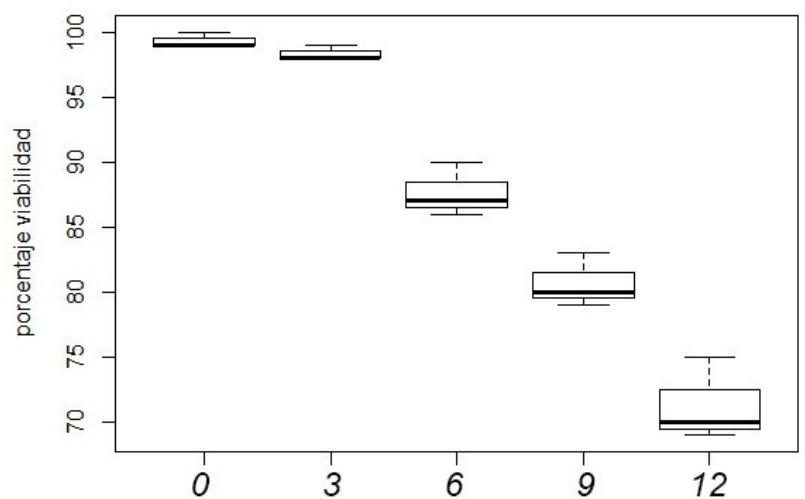

B) tales, edad, contenido de humedad y de las condiciones de manejo y almacenamiento (Bradford 2007, Manjarrez-Juárez et al. 2017). La disminución de la viabilidad puede deberse a la falta de integridad de las membranas celulares de las semillas, por disminución de fosfolípidos, carbohidratos y proteínas, lo que provoca que la estructura y funcionamiento de las semillas se modifique, y se generen cambios en la permeabilidad, pérdida de fluidez e integridad (Melo y Cuamatzi 2008), o por el tipo de testa, que determina si estas pueden ser conservadas por cortos o largos periodos de tiempo (Kameswara-Rao et al. 2007).

La germinación se registró a los 15 y $25 \mathrm{~d}$ después de la siembra para $C$. vitifolium y $Q$. funebris respectivamente (Figura $2 \mathrm{~A}$ y $2 \mathrm{~B}$ ), el porcentaje de germinación fue afectado de forma significativa $(p<0.000)$ por el tiempo de almacenamiento, donde las semillas de $C$. vitifolium a los cero meses de almacenamiento tuvieron un $98.6 \%$ de germinación, mientras que para $Q$. Funebris fue del $96.6 \%$. A 12 meses de almacenamiento el porcentaje de semillas germinadas disminuyó al 71.3 y $61.3 \%$ para $C$. vitifolium y $Q$. funebris (Figura $3 A$ y 3B). Al respecto Duarte et al. (2014) menciona que existe una relación entre la presencia de la testa y los bajos valores de germinación que se registran en los ensayos de germinación, dependiendo el tipo de semilla.

Las variables germinativas determinadas en $C$. vitifolium y $Q$. funebris indican que las semillas de ambas especies presentan condiciones óptimas para la germinación en almacenamiento a cero meses, mismo que disminuye a los 12 meses, al respecto Soplin (1988) y Navarro y Deméneghi (2007), menciona que las condiciones de maduración, la temperatura y humedad en que las semillas permanecen, influyen en la viabilidad y capacidad ger- 

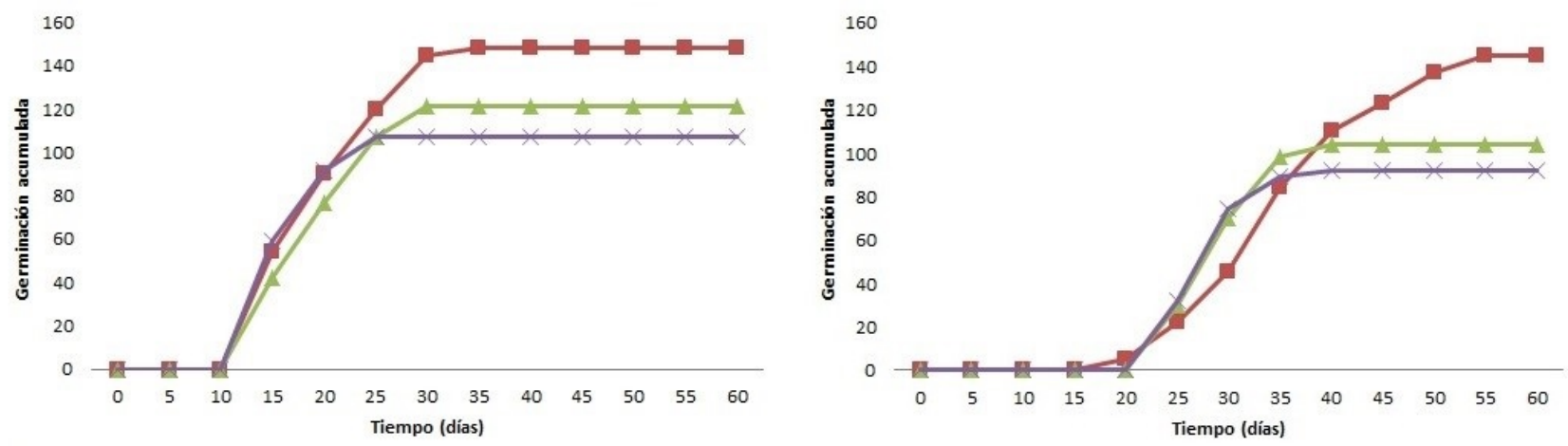

A

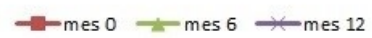

B

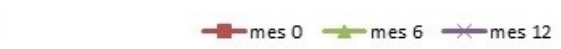

Figura 2. Germinación acumulada en semillas de A) C. vitifolium, y B) Q. funebris.
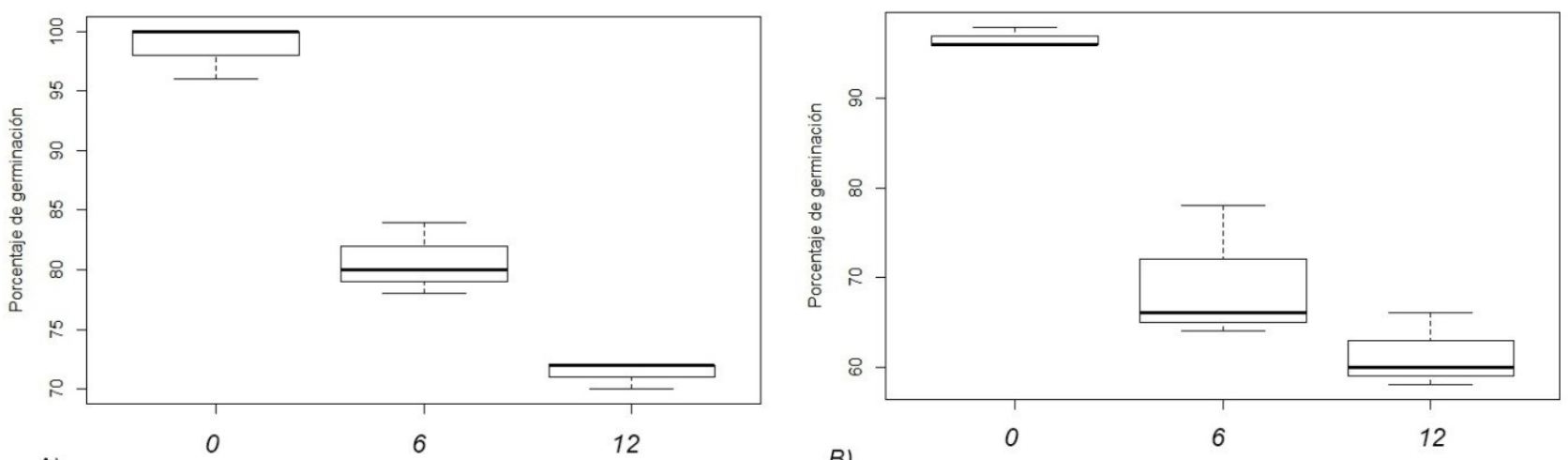

A)

Figura 3. Porcentaje de germinación por tiempo de almacenamiento A) C. vitifolium, presenta porcentajes de germinación de $0=98.6 \%, 6=80 \%$ y $12=71.3 \%$ y B) $Q$. funebris con la presente germinación $0=96.6 \%, 6=69.3 \%$ y $12=61.3 \%$.

Tabla 2. Variables evaluadas en la prueba de germinación en semillas de C. vitifolium y $Q$. funebris en diferentes tiempos de almacenamiento.

\begin{tabular}{ccccccc}
\hline Especie & Tiempo (meses) & $\mathrm{T}$ & IG & MG & CV & Mann-Whitney \\
\hline \multirow{3}{*}{ C. vitifolium } & 0 & 17.94 & 80.38 & 2.46 & 4.72 & $\mathrm{a}$ \\
& 6 & 20.73 & 65.38 & 2.05 & 4.82 & $\mathrm{~b}$ \\
\hline \multirow{2}{*}{ Q. funebris } & 12 & 21.18 & 49.23 & 1.78 & 5.57 & $\mathrm{c}$ \\
& 0 & 29.40 & 137.05 & 2.41 & 2.71 & $\mathrm{a}$ \\
& 6 & 30.48 & 81.28 & 1.73 & 3.28 & $\mathrm{~b}$ \\
\hline
\end{tabular}

Tiempo promedio de germinación ( $T$ ), índice de germinación (IG), velocidad de germinación (MG), coeficiente de germinación (CV). Las letras representan las diferencias entre meses para cada especie.

minativa, la cual disminuye con el tiempo. Por otro lado, el vigor de las semillas, se evalúa cuando hay una reducción en la germinación o producción de plántulas, mediante algunas variables del proceso germinativo, como tiempo de germinación $(T)$, índice de germinación (IG), velocidad de germi- nación (MG) y coeficiente de germinación (CV) (Ferguson 1995). El almacenamiento es un factor que influye en la viabilidad y la germinación de las semillas de $C$. vitifolium y $Q$. funebris, que tuvieron una pérdida de viabilidad y germinación con el tiempo de almacenamiento. 


\section{LITERATURA CITADA}

Bidwell RGS (2000) Fisiología vegetal. AGT Editores. México. 784p.

Bradford KJ, Nonogaki H (2007) Seed development, dormancy and germination. Blackwell Publishing. Oxford. UK. 39p.

Cedano-Maldonado M, Villaseñor-Ibarra L (2004) Usos y nombre comunes de las especies de Cochlospermaceae en México. Etnobiología 4: 73-88.

Challenger A, Soberón J (2008) Los ecosistemas terrestres. En: Comisión nacional para el conocimiento y uso de la biodiversidad (Conabio) (ed). Capital natural de México: Conocimiento actual de la biodiversidad. Vol. I. CONABIO. México. pp: 87-108.

Duarte E, Acivo E, Sansberro P, Luna C (2014) Efecto de la testa sobre la germinación de semillas de Handroanthus heptaphyllus tras distintos tiempos de almacenamiento. Ciencias Agronómicas 29: 2935.

Ferguson J (1995) An introduction to seed vigour testing. In: International Seed Testing Association (ed) Seed vigour testing. Copenhagen, Zurich. pp: 1-9.

Franca-Neto J, Krzyznowski FC, Costa NP (1998) El test de tetrazolio en semillas de soja. Embrapa. Londrina, Brasil. 72p.

Gaston KJ (2000) Global patterns in biodiversity. Nature 405: 220-227.

Gentil DFO (2001) Conservação de sementes do cafeeiro: resultados discordantes ou complementares. Bragantia 3: 149-154.

González-Zertuche HML, Orozco-Segovia A (1996) Métodos de análisis de datos de la germinación de semillas, un ejemplo: Manfreda brachystachya. Boletín de la Sociedad Botánica de México 58: 37-52.

Hartmann HT, Kester DE (2001) Propagación de Plantas y Principios Básicos. CECSA. México. 760p.

INEGI (2013) Conociendo México. 3a Edición. INEGI. México. 86p.

ISTA (2005) Reglas Internacionales para Ensayos de Semillas. Ensayo topográfico al Tetrazolio. Secretaría de Estado de Agricultura y Ganadería. España. https://www.seedtest.org/en/home.html. Fecha de consulta 02 de julio de 2016.

ISTA (2010) Reglas del ISTA. Septiembre de 2010. http://www.analisisdesemillas.com.ar/index.php?option= com content\&task =view\&id=15\&ltemid=31. Fecha de consulta 02 de Julio de 2016.

Kameswara-Rao N, Hanson J, Ehsan-Dulloo M, Ghosh K, Nowell D, Larinde M (2007) Manual para el manejo de semillas en bancos de germoplasma. Manuales para bancos de germoplasma. Biodiversity international. USA. 165p.

Manjarrez-Juárez FJ, Díaz-Huacuz R, Carballo-Carballo A, Estrada-Gómez A, Vaquera-Huerta H, AcostaGallegos JA, et al. (2017) Efecto del tiempo de almacenamiento sobre la calidad de semilla de canola. Revista Mexicana de Ciencias Agrícolas 8: 933-948

Maldonado-Peralta MA, García SG, García-Nava JR, Ramírez-Herrera C, Hernández-Livera A, Valdez-Carrazco JM, Torres-Corona T y Cetina-Alcalá VW (2016) Seed viability and vigour of two nanche species (Malpighia mexicana and Byrsonima crassifolia). Seed Science \& Technology 44: 1-9.

Melo RV, Cuamatzi T (2008) Bioquímica de los procesos metabólicos. 2a ed. Ed. Reverté. México. 406p. 
Navarro MC, Deméneghi AP (2007) Germinación de semillas y efecto de las hormonas en el crecimiento de Mammillaria pectinifera. Zonas Áridas 11: 233-239

Nkang A (2002) Carbohydrate composition during seed development and germination in two sub-tropical rainforest tree species (Erythrina caffra and Guilfoylia monostylis). Journal Plant Physiology 159: 473483.

Pennington TD, Sarukhán J (2005) Árboles tropicales de México. 3da Edición. Editorial Universidad Nacional Autónoma de México. Fondo de Cultura Económica. México. 521p.

Pérez-García F, Pita-Villamil JM (2001) Viabilidad, vigor, longevidad y conservación de semillas. Ministerio de Agricultura, Pesca y Alimentación. Madrid, España. 16p.

Ríos-García CA, Orantes-García C, Moreno-Moreno RA, Farrera-Sarmiento O (2016) Viabilidad y germinación de semillas de jopi (Ochroma pyramidale (Cav. Ex Lam.) Urb.) (Malvaceae). Lacandonia 10: 7-11.

Rzedowski J (1991) Diversidad y orígenes de la flora fanerogámica de México. Acta Botánica Mexicana 14: 3-21.

Sánchez-Arellano JG, Parra-Galindo MA, Silvas-Olivas MF, Pedroza-Pérez D (2011) Efecto de la temperatura y tiempo de almacenamiento sobre la viabilidad en semillas de zámota (Coursetia glandulosa, Gray). Biotecnia 13: 36-40.

Soplin H (1988) Factores que afectan el potencial de almacenamiento de las semillas. In: INIA (ed). Monografía: Conservación y uso de los recursos genéticos. Perú. http://www.inia.gob.pe/. Fecha de consulta 05 de julio de 2016.

Velázquez A., Mas JF, Díaz GJR, Mayorga SR, Alcántara PC, Castro R, Palacio JL (2002) Patrones y tasas de cambio de uso de suelo en México. Gaceta Ecológica 62: 21-37 
